\title{
ABORDAGEM DAS DISFUNÇÕES SEXUAIS: O USO DE LEPIDIUM MEYENII E TRIBULUS TERRESTRIS
}

\author{
APPROACH TO SEXUAL DYSFUNCTIONS: THE USE OF LEPIDIUM MEYENII \\ AND TRIBULUS TERRESTRIS
}

\author{
Edcleidson Rabelo da Cruz \\ Juliana Azevedo da Paixão \\ Yuri Gabriel Batista Silva
}

\begin{abstract}
RESUMO: Objetivo : realizar uma revisão da literatura sobre a abordagem das disfunções sexuais com o auxílio da Lepidium meyenii e Tribulus terrestres .Métodos : Trata-se de uma de uma pesquisa bibliográfica, descritiva, com abordagem qualitativa. Resultados: $\mathrm{O}$ resultado total da investigação foi de 70 artigos. Foram excluídos 13 artigos repetidos. Restou o total de 57 artigos, dos quais foram excluídos os que não continham o texto disponível e também os que não estavam relacionados às Disfunções Sexuais humanas . Assim, foram obtidos I6 artigos . Foram descartados os artigos que não possuíam texto completo disponível ou que seu conteúdo não relacionava com a temática .Considerações finais: Com base nos estudos discutidos é possível Tribulus terrestris consiste em uma planta com grandes potencialidades para o manejo da disfunção sexual e outros sintomas relacionados à menopausa, sendo assim, pode ser um tratamento alternativa ou complementar.
\end{abstract}

Palavras-chave : Disfunção sexual. Tribulus terrestris.Lepidium meyenii. Saúde Sexual.

ABSTRACT: Objective: to carry out a literature review on the approach of sexual dysfunctions with the help of Lepidium meyenii and terrestrial Tribulus. Methods: This is a bibliographic, descriptive research with a qualitative approach. Results: The total result of the investigation was 70 articles. 13 repeated articles were excluded. A total of 57 articles remained, of which those that did not contain the available text and those that were not related to human Sexual Dysfunctions were excluded. Thus, 16 articles were obtained. Articles that did not have the full text available or that their content did not relate to the theme were discarded. Conclusion: Based on the studies discussed, it is possible Tribulus terrestris is a plant with great potential for the management of sexual dysfunction and other symptoms related to menopause, therefore, can be an alternative or complementary treatment.

Keywords: Sexual dysfunction. Tribulus terrestris. Lepidium meyenii. Sexual health

\section{INTRODUÇÃO}

A sexualidade engloba toda a vida de uma pessoa, e sua abordagem vem sendo 
cada vez mais tido atenção. A Organização Mundial de Saúde entende que a sexualidade consiste em um dos elementos que favorecem a melhor qualidade de vida, sendo um pressuposto central que atravessa por toda a vida do ser humano. Todas as pessoas devem ter a garanta do direito à saúde sexual, que pode ser definida como um estado de bem-estar emocional, físico e social relacionado à sexualidade. Contudo, para manutenção da boa saúde sexual, é relevante a interação de fatores biológicos, psicológicos e sociais. Sendo assim, a qualidade da saúde sexual pode influenciar de modo significativo no bem-estar e na qualidade de vida de uma pessoa (QUEIROZ et al.,2015).

Nos últimos anos é possível observar o avanço na abordagem da disfunção sexual masculina por meio de novas e mais efetivas alternativas de tratamento para a disfunção erétil. Ademais, fatores ligados à integridade biológica e psicológica favorecem o desenvolvimento dos transtornos sexuais masculinos, fatores sociais também estão na lista da etiologia desses transtornos(SANTOS et al.,2015).

É oportuno comentar que a discussão sobre disfunção sexual masculina, favoreceu uma maior abertura para o debate acerca da sexualidade feminina. Com base nisso, a importância da satisfação sexual feminina tem sido cada vez mais valorizada na sociedade atual, o que permitiu que saúde sexual e reprodutiva avançasse nestes últimos anos, sobretudo, no que se refere à saúde sexual feminina (RIBEIRO et al.,2016).

Em contrapartida, a resposta sexual feminina é complexa e ainda não foi totalmente elucidada.Constantemente, emergem controvérsias sobre problemas sexuais femininos e as suas formas de abordagem. No entanto, o reconhecimento de que o distúrbio sexual feminino é uma situação prevalente na atual sociedade, que impacta na qualidade de vida da mulher. Vale explicar que o desejo sexual hipoativo é o mais prevalente das disfunções sexuais, e está relacionado à qualidade de vida da mulher. Diversos fatores biológicos podem colaborar para o aparecimento do desejo sexual hipoativo tais quais: fatores anatômicos, neuronais, endócrinos e vasculares. Sendo assim, medicamentos são testados para o enfrentamento desse distúrbio, com o objetivo promover uma melhor qualidade de vida dessas mulheres (BARRETO et al.,2018). 
Existe a possibilidade de abordagem das disfunções sexuais por meio da utilização de plantas medicinais, estas tem sido utilizada com finalidade terapêutica há milhares de anos, devido as propriedades afrodisíacas. O uso de dos fitoterápicos traz como principais vantagens apresentar alto índice de efeitos terapêuticos com baixa incidência de efeitos colaterais (ZENI et al.,2017).

Existem finalidades para utilização de fitoterápicos são diversas e há até aqueles implicados potencialmente nas disfunções sexuais humanas. Entre as plantas mais conhecidas para o manejo das disfunções sexuais estão: Tribulus da terrestres, Lepidium meyenii, Panax ginseng, Ginkgo biloba, Pausinystalia johimbe. Este estudo tratará Tribulus terrestres e, Lepidium meyenii(COSTA et al.,2020).

Nesta direção, vale citar que o Tribulus terrestres consiste em uma planta originária da Índia, muito utilizada como um estimulante sexual natural pela medicina tradicional da China, Índia e Grécia. O Tribulus é capaz de elevar as concentrações séricas de testosterona endógena, o que justifica os efeitos demonstrados na função erétil, contudo, ainda não foi elucidado como o Tribulus exerce esse aumento (FERNANDES et al.,2019).

Além disso, o Tribulus terrestris é composto principalmente por esteróides, saponinas, flavonóides e alcalóides. As saponinas hidronizadas transformam-se em sapogeninas esteroidais, apresentando propriedades antiespasmódicas e diuréticas, aumentam a produção de hormônio luteinizante (LH), testosterona, estrogênio e outros esteroides (SOUSA et al.,2019).

A maca peruana, Lepidium meyenii, consiste em uma planta nativa da região dos Andes, muito conhecida e consumida em países como Peru, Bolívia, Equador e Venezuela. Sua parte comestível é a raiz, constituída por substâncias com propriedades antioxidantes e metabólicas, relacionado à presença de fenóis e flavonoide. A Lepidium meyenii é utilizada para aumentar a vitalidade, no stress, para promover a libido, aumentar a fertilidade e o desempenho sexual em homens e mulheres (WANG,2019).

O estudo o tema justifica-se porque as plantas medicinais e os medicamentos fitoterápicos são amplamente utilizados no Brasil como alternativa terapêutica, sendo assim, o presente estudo justifica-se pela possibilidade de discutir sobre o manejo das 
disfunções sexuais com o auxílio da Lepidium meyenii e Tribulus terrestres. Sendo o profissional farmacêutico importante na difusão de informações relativas ao uso de plantas medicinais, tornam-se relevante a realização de estudos sobre este tema pela intencionalidade de levar à comunidade acadêmica informações sobre o assunto.

Diante do exposto este estudo tem como objetivo geral : realizar uma revisão da literatura sobre a abordagem das disfunções sexuais com o auxílio da Lepidium meyenii e Tribulus terrestres. E como objetivos específicos: avaliar a eficácia da Lepidium meyenii e Tribulus terrestres no tratamento da disfunção sexual humana;descrever a composição química da Lepidium meyenii e Tribulus terrestres; discutir as propriedades farmacológicas da Lepidium meyenii e Tribulus terrestres.

\section{METODOLOGIA}

Trata-se de uma de uma pesquisa bibliográfica, integrativa, descritiva, com abordagem qualitativa. As fontes bibliográficas foram identificadas através das bases de dados Scientific Electronic Library Online (SCIELO), o qual disponibiliza na Internet, os textos completos de artigos de revistas científicas e no Literatura Latino Americana e do Caribe em Ciência da Saúde (LILACS), que é índice bibliográfico da literatura relativa às ciências da saúde, publicada nos países da América Latina e Caribe. Public Medline (PubMed), CAPES Periódicos, bem como livros que tratam sobre a temática.

Para a busca dos artigos foram utilizadas as seguintes palavras-chave: disfunção sexual, Tribulus terrestris, Lepidium meyenii, ,Saúde sexual. Quanto aos critérios de inclusão foram incluídos artigos que abordaram os objetivos do presente trabalho; artigos publicados entre os anos de 2015 à 2021; artigos em inglês, português; artigos delimitados como revisão de literatura, estudos populacionais, estudos clínicos randomizado. E os critérios de exclusão foram excluídos atigos que não sejam disponibilizados de forma completa online; artigos que não se enquadram nos parâmetros de inclusão. Após a seleção dos artigos, os estudos foram organizados através da caracterização das publicações, contemplando aspectos gerais sobre os artigos: autoria, ano de publicação, periódico, área do conhecimento. 


\section{RESULTADOS E DISCUSSÃO}

O resultado total da investigação foi de 70 artigos. Foram excluídos 13 artigos repetidos. Restando o total de 57 artigos, dos quais foram excluídos os que não continham o texto disponível e também os que não estavam relacionados às Disfunções Sexuais humanas. Assim, foram obtidos 16 artigos. Foram descartados os artigos que não possuíam texto completo disponível ou que seu conteúdo não relacionava com a temática. No quadro I, estão descritos os estudos que mencionaram Tribulus terrestres e Lepidium meyenii no tratamento da disfunção sexual.

Quadro I. Estudos que mencionaram Tribulus terrestres e Lepidium meyenii no tratamento da disfunção sexual.

\begin{tabular}{|c|c|c|c|}
\hline AUTORES / ANO & TÍTULO & METODOLOGIA & $\begin{array}{c}\text { PRINCIPAIS } \\
\text { RESULTADOS }\end{array}$ \\
\hline $\begin{array}{l}\text { GUAZZELLI } \\
\text { (2014). }\end{array}$ & $\begin{array}{l}\text { Estudo dos efeitos do } \\
\text { Tribulus terrestris e } \\
\text { da tibolona em } \\
\text { mulheres com } \\
\text { disfunção do desejo } \\
\text { sexual após a } \\
\text { menopausa/Study of } \\
\text { the effects of Tribulus } \\
\text { terrestris and tibolone } \\
\text { in postmenopausal } \\
\text { women with sexual } \\
\text { desire dysfunction. }\end{array}$ & $\begin{array}{l}\text { Estudo prospectivo, } \\
\text { randomizado. }\end{array}$ & $\begin{array}{l}\text { Quanto ao padrão do } \\
\text { Desempenho } \\
\text { Sexual, inicialmente } \\
\text { todos os Grupos } \\
\text { apresentavam um } \\
\text { padrão desfavorável } \\
\text { a regular, e, ao final } \\
\text { do estudo, o Grupo } \\
\text { Controle manteve } \\
\text { o mesmo padrão, o } \\
\text { Grupo Tribulus } \\
\text { passou a apresentar } \\
\text { um padrão regular a } \\
\text { bom, e o Grupo } \\
\text { Tibolona, um } \\
\text { padrão bom a } \\
\text { excelente. }\end{array}$ \\
\hline GONZALES & (Lepidium & Revisão narrativa. & $\mathrm{O}$ efeito não é por \\
\hline
\end{tabular}




\begin{tabular}{|c|c|c|c|}
\hline al.(2014). & $\begin{array}{l}\text { meyenii Walp), una } \\
\text { revisión sobre sus } \\
\text { propiedades } \\
\text { biológicas. }\end{array}$ & & $\begin{array}{l}\text { meio de alterações } \\
\text { hormonais, mas sim } \\
\text { pós-receptor. }\end{array}$ \\
\hline SOUZA(2015). & $\begin{array}{l}\text { Avaliação dos efeitos } \\
\text { do Tribulus terrestris } \\
\text { em mulheres na pós- } \\
\text { menopausa com } \\
\text { distúrbio do desejo } \\
\text { sexual hipoativo. }\end{array}$ & $\begin{array}{l}\text { Ensaio clínico, } \\
\text { prospectivo, } \\
\text { randomizado, duplo- } \\
\text { cego, placebo- } \\
\text { controlado. }\end{array}$ & $\begin{array}{l}\text { O uso do Tribulus } \\
\text { terrestris em } \\
\text { comparação ao } \\
\text { placebo com base no } \\
\text { questionário Índex } \\
\text { da função sexual } \\
\text { feminina (FSFI) } \\
\text { mostrou melhora } \\
\text { significativa no } \\
\text { domínio } \\
\text { lubrificação. } \\
\text { Quando comparado } \\
\text { o uso do Tribulus } \\
\text { terrestris com o } \\
\text { placebo a partir do } \\
\text { questionário } \\
\text { Quociente sexual } \\
\text { feminino (QS-F), } \\
\text { observaram-se } \\
\text { significativa } \\
\text { melhora } \\
\text { domínios HSDD, } \\
\text { excitação/lubrificaç } \\
\text { ão, dor e anorgasmia } \\
\text { nas mulheres que } \\
\text { usaram T. terrestres. }\end{array}$ \\
\hline
\end{tabular}




\begin{tabular}{|c|c|c|c|}
\hline $\begin{array}{l}\text { DORDING et al.( } \\
\text { 2015). }\end{array}$ & $\begin{array}{l}\text { A double-blind pla- } \\
\text { cebo-controlled trial } \\
\text { of maca root as treat- } \\
\text { ment for antidepres- } \\
\text { sant-induced sexual } \\
\text { dysfunction in } \\
\text { women. }\end{array}$ & $\begin{array}{l}\text { Ensaio clínico, } \\
\text { randomizado. }\end{array}$ & $\begin{array}{l}\text { As taxas de remissão } \\
\text { ao final do } \\
\text { tratamento foram } \\
\text { maiores para o grupo } \\
\text { maca do que para o } \\
\text { grupo placebo, com } \\
\text { base na obtenção de } \\
\text { uma pontuação total } \\
\text { ASEX } \leq \text { Io (9,5\% } \\
\text { para maca versus } \\
4,8 \% \text { para placebo), } \\
\text { atingindo uma } \\
\text { pontuação MGH- } \\
\text { SFQ } \leq \text { I2 (30,o\% para } \\
\text { maca versus 20,o\% } \\
\text { para placebo) } \\
\text { atingindo uma } \\
\text { pontuação MGH- } \\
\text { SFQ } 8 \text { (9,5\% para } \\
\text { maca versus } 5,0 \% \\
\text { para placebo). }\end{array}$ \\
\hline $\begin{array}{l}\text { POSTIGO et } \\
\text { al.(2oi6). }\end{array}$ & $\begin{array}{l}\text { Assessment of the ef- } \\
\text { fects of Tribulus ter- } \\
\text { restris on sexual func- } \\
\text { tion of menopausal } \\
\text { women. }\end{array}$ & $\begin{array}{c}\text { Ensaio clínico, } \\
\text { prospectivo, } \\
\text { randomizado. }\end{array}$ & $\begin{array}{l}\text { Após 9o dias de } \\
\text { tratamento, nas } \\
\text { doses utilizadas, } \\
\text { descobrimos que o } \\
\text { Tribulus terrestris é } \\
\text { eficaz no tratamento } \\
\text { de problemas } \\
\text { sexuais entre }\end{array}$ \\
\hline
\end{tabular}




\begin{tabular}{|c|c|c|c|}
\hline & & & $\begin{array}{l}\text { mulheres na } \\
\text { menopausa. }\end{array}$ \\
\hline CHEN;FAN( 2017). & $\begin{array}{l}\text { The nutritional com- } \\
\text { position of maca in } \\
\text { hypocotyls (Lepid- } \\
\text { ium meyenii Walp.) } \\
\text { cultivated in different } \\
\text { regions of China. }\end{array}$ & Ensaio clínico. & $\begin{array}{l}\text { Em geral, as áreas de } \\
\text { cultivo e o tipo de } \\
\begin{array}{lr}\text { cor da maca } \\
\text { afetaram certas } \\
\text { composições }\end{array} \\
\text { substâncias } \\
\text { secundárias }\end{array}$ \\
\hline $\begin{array}{l}\text { SIDDIQUI; BAIG; } \\
\operatorname{AHMAD(~2017).~}\end{array}$ & $\begin{array}{l}\text { Phytochemical and } \\
\text { biological assess- } \\
\text { ments on Lipidium } \\
\text { meyenii (maca) and } \\
\text { Epimidium sagitta- } \\
\text { tum (horny goat } \\
\text { weed). }\end{array}$ & Ensaio clínico. & $\begin{array}{l}\text { No geral, esses } \\
\text { resultados sugerem } \\
\text { que o LM é } \\
\text { altamente remédio } \\
\text { eficaz para o } \\
\text { tratamento a } \\
\text { impotência e reduzo } \\
\text { estresse e } \\
\text { depressão, por causa } \\
\text { do duplo efeito ES } \\
\text { não só sugerido } \\
\text { como um } \\
\text { medicamento } \\
\text { ansiolítico, } \\
\text { também eficaz no } \\
\text { distúrbio hormonal. }\end{array}$ \\
\hline $\begin{array}{l}\text { FATIMA; } \\
\text { SULTANA(2017). }\end{array}$ & $\begin{array}{l}\text { Efficacy of Tribulus } \\
\text { terrestris L.(fruits) in } \\
\text { menopausal transi- } \\
\text { tion symptoms: A } \\
\text { randomized placebo } \\
\text { controlled study }\end{array}$ & $\begin{array}{l}\text { Ensaio clínico, } \\
\text { prospectivo, } \\
\text { randomizado. }\end{array}$ & $\begin{array}{l}\text { O Tribulus foi mais } \\
\text { eficaz do que o } \\
\text { placebo no alívio dos } \\
\text { sintomas de } \\
\text { transição da } \\
\text { menopausa e pode }\end{array}$ \\
\hline
\end{tabular}




\begin{tabular}{|c|c|c|c|}
\hline & & & $\begin{array}{l}\text { oferecer uma } \\
\text { alternativa segura à } \\
\text { terapia de reposição } \\
\text { hormonal. }\end{array}$ \\
\hline SOUSA(2019). & $\begin{array}{l}\text { Tribulus terrestris Linn } \\
\text { como tratamento da } \\
\text { sintomatologia da } \\
\text { menopausa: uma } \\
\text { revisão sistemática. }\end{array}$ & Revisão sistemática. & 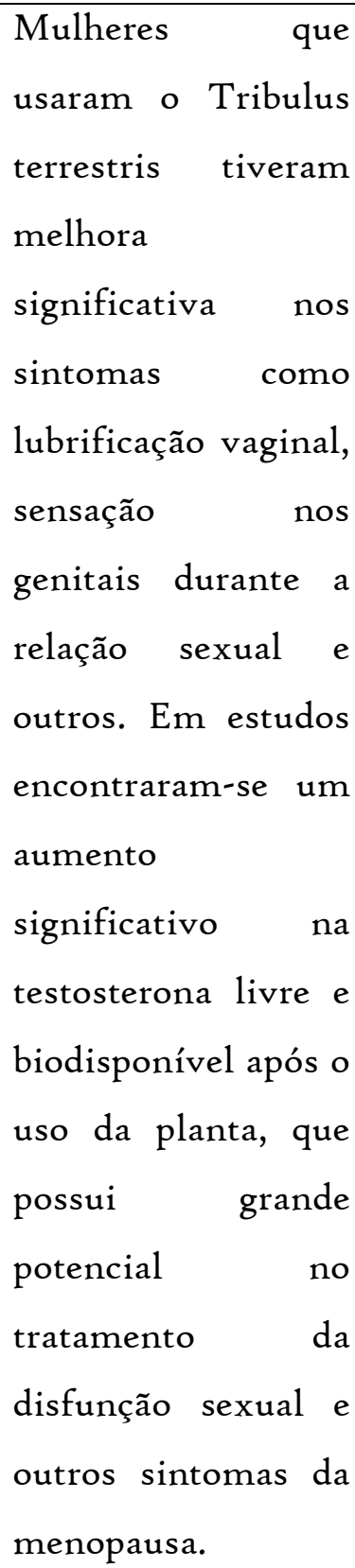 \\
\hline PERES et al.(2020). & $\begin{array}{l}\text { Medicinal effects of } \\
\text { Peruvian maca } \\
\text { (Lepidium meyenii): a } \\
\text { review. }\end{array}$ & Revisão de literatura. & $\begin{array}{ll}\text { A maca peruana é } \\
\text { destaque } \\
\text { literatura } \\
\text { tratamento no }\end{array}$ \\
\hline
\end{tabular}




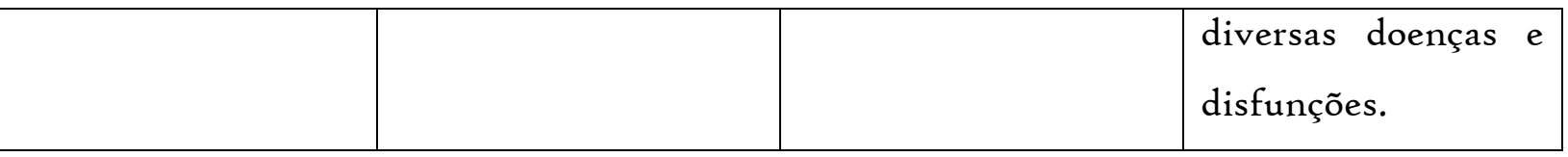

Fonte : Autoria própria (2021)

$O$ estilo de vida pode interferir de forma benéfica ou prejudicial no funcionamento do organismo. A associação de fatores nutricionais inadequados possui um impacto na saúde sexual e reprodutiva em homens e mulheres. Um fator que pode interferir é a obesidade e está associada a níveis séricos de testosterona e hormônio luteinizante $(\mathrm{LH})$ mais baixos, taxas elevadas de oligospermia ou azoospermia, e diminuição do volume de ejaculação, concentração de esperma e contagem total de esperma (BARRETO et al.,2018).

No que concerne ao Afrodisíaco Queiroz et al. (2015), Ribeiro et al. (2016), explicaram em seus estudos que consiste em qualquer alimento ou medicamento que desperta o instinto sexual, induz desejo e aumenta o prazer e o desempenho sexual, os produtos afrodisíacos são produtos que na rotulo descreve que desperta ou aumenta a sexualidade, desejo ou que vai melhorar o desempenho sexual.

Ribeiro et al. (2016), (2009), caracterizou que não há um consenso universal acerca da classificação dos afrodisíacos, mas estes podem ser denominados de afrodisíacos naturais, os que são originados de vegetais, plantas, ou sintéticos que provem de drogas farmacológicas e que os afrodisíacos podem, também, ser classificados por seu modo de ação em três tipos: aqueles que aumentam a libido, a potência ou o prazer sexual.

Zeni et al. (2017), discutiram que diversas substâncias de origem animal e vegetal têm sido usadas em medicamentos populares de diferentes culturas para energizar, vitalizar e melhorar a função sexual e o desempenho físico e que uma refeição para ser considerada afrodisíaca precisa ter no mínimo um ingrediente com essa finalidade. Seu uso ainda pode ser feito através de pílulas ou como condimento.

O Tribulus terrestres e Lepidium meyenii são exemplos de um número muito limitado de afrodisíacos naturais que tem passado por investigações científicas rigorosas para explorar suas propriedades afrodisíacas (ZENI et al.,2017).

No estudo realizado por Postigo et al .(2016), cujo objetivo foi estudar os efeitos 
do Tribulus terrestris na função sexual em mulheres na menopausa, por meio de um ensaio clínico prospectivo, randomizado, duplo-cego e controlado por placebo que incluiu 6o mulheres na pós-menopausa com disfunção sexual.

Como achado mais relevante o estudo de Postigo et al (2016) evidenciou que no grupo placebo, 20\% das mulheres relataram uma melhora na experiência sexual e desejava continuar usando o medicação, 23,3\% não perceberam mudanças em suas relações sexuais experiência, mas desejava continuar tomando a medicação, e 56,7\% relataram que sua experiência sexual não mudou e não queriam continuar com a medicação. No grupo Tribulus, $80 \%$ relataram uma melhora em seus experiência sexual e desejavam continuar tomando a medicação, I0\% relataram nenhuma mudança, mas gostariam de continuar usando a medicação, e Io\% relataram nenhuma mudança e não deseja continuar a medicação.

Souza (2015), realizou um estudo semelhante ao de Postigo et al .(2016) que avaliou os efeitos do Tribulus terrestris em mulheres com disfunção do desejo sexual hipoativo na pós-menopausa, avaliando a sua eficácia no tratamento do distúrbio do desejo sexual hipoativo (HSDD) e sua influência nos níveis séricos da testosterona.

O estudo de Souza (2015), trouxe como aspecto mais relevante que Tribulus terrestris é uma planta com grandes potencialidades no tratamento da disfunção sexual e outros sintomas relacionados à menopausa, dessa forma, pode vir a ser uma terapêutica alternativa ou complementar. Contudo, estudos mais precisos e munidos de avaliação clínica são necessários para uma análise mais robusta e confiável da atividade de Tribulus terrestris.

Assim como Postigo et al .(2016), Guazzelli et al.(2014), estudaram os efeitos do Tribulus terrestris e da Tibolona em mulheres com disfunção do desejo sexual após a menopausa, através de um estudo prospectivo, randomizado realizado com 66 mulheres após a menopausa com disfunção do desejo sexual. Como achado mais relevante e em concordância com Postigo et al (2016), os Grupos Controle e Tribulus todas concluíram o estudo; no Grupo Tibolona quatro não concluíram, sendo três por efeitos adversos e que nos Grupos Tribulus e Tibolona houve melhora significante após o tratamento em todos os domínios avaliados. No grupo Controle houve melhora estatisticamente significante nos aspectos desejo e interesse sexual, e capacidade 
de excitação; e a pontuação total no QS-F apresentou uma piora estatisticamente significante.

O estudo de Guazzelli et al. (2014), também mostrou que o padrão do Desempenho Sexual, inicialmente todos os Grupos apresentavam um padrão desfavorável a regular, e, ao final do estudo, o Grupo Controle manteve o mesmo padrão, o Grupo Tribulus passou a apresentar um padrão re-gular a bom, e o Grupo Tibolona, um padrão bom a excelente e que $\mathrm{O}$ Tribulus terrestris e a Tibolona apresentaram resposta favorável no tratamento de mulheres com disfunção.

Sousa e Lima (2019), analisou através de uma revisão sistemática a capacidade de Tribulus terrestris em promover o aumento dos níveis hormonais como mecanismo para alívio dos sintomas da menopausa. Sousa e Lima (2017), observaram que o Tribulus terrestris foi melhor que o placebo para reduzir sintomas somáticos, psicológicos e urogenitais e que as mulheres que usaram o Tribulus terrestris tiveram melhora significativa nos sintomas como lubrificação vaginal, sensação nos genitais durante a relação sexual e outros e que existe um aumento significativo na testosterona livre e biodisponível após o uso da planta, que possui grande potencial no tratamento da disfunção sexual e outros sintomas da menopausa. Quanto aos efeitos adversos nos estudos foram citados uma maior incidência de diarreia, nervosismo, tontura, náuseas, rubor facial, náusea.

A Maca Peruana (Lepidium meyenii) pertence à família Brassicaceae e cresce na região central dos Andes do Peru acima de 4000 metros de altitude, onde é cultivado por mais de 2.000 ano. A maca é um extrato vegetal, tem sido relatada nos estudos como um produto capaz de melhorar o desejo sexual, devido a suas atividades espermatogênicas e de aumento da fertilidade, associado aos fitoesteróis ou fitoestrogênios presentes na maca peruana (SIDDIQUI; BAIG;AHMAD, 2017).

Os hipocótilos secos da maca peruana são aproximadamente 13-16\% de proteína e são ricos em aminoácidos essenciais. $\mathrm{O}$ hipocótilo fresco contém $80 \%$ de água e grandes quantidades de ferro e cálcio. Uma descrição mais completa da composição da maca seca mostra Io,2\% de proteínas, 59\% de carboidratos, 2,2\% de lipídios e 8,5\% de fibras.

Quando usados como um produto alimentar, a maca peruana é frequentemente 
fervida e bebida como um suco, devido à forma de armazenamento de hipocótilos secos ser muito difícil de mastigar. O uso moderno da maca peruana tende a ser em cápsulas(WANG; ZHU,2019).

Dording et al. (2015), demonstraram que a raiz de maca pode ser um tratamento eficaz para disfunção sexual induzida por antidepressivos (AISD) em mulheres, além disso, evidenciou que as taxas de remissão da disfunção sexual ao final do tratamento foram maiores para a maca do que para o grupo de placebo e que essa diferença parece ser impulsionada por uma coorte de mulheres pós-menopáusicas.

Dording et al. (2015), relatou que estudo é limitado por vários fatores, tamanho da amostra foi relativamente pequeno e que o estudo contou com o autorrelato do paciente no domínio do orgasmo, que é subjetivo e pode estar sujeito a preconceitos e imprecisões.

Gonzales et al. (2014), realizaram um estudo que evidenciou que em homens normais há uma melhora do desejo sexual após 8 semanas de administração, que foi considerado um longo tempo para um tratamento para melhorar o desejo sexual, mas em contrapartida, o uso de extrato de maca melhorou o desejo sexual em atletas do sexo masculino após duas semanas de tratamento .

No estudo de Gonzales et al. (2014), foi mostrando ainda que no sexo masculino a Maca aumenta o desejo sexual, a produção, No estudo de Gonzales et al. (2014), foi mostrando ainda que no sexo masculino a Maca aumenta a libido, a síntese de espermatozóides e melhora a disfunção erétil de intensidade leve, e no sexo feminino foi observada uma diminuição dos sintomas de desconforto da menopausa, assim como melhora da disfunção sexual associada ao climatério.

Os estudos mostraram que a Maca reduz os sintomas psicológicos, incluindo ansiedade e depressão, além de diminuir as medidas de disfunção sexual em mulheres na pós-menopausa independente da atividade estrogênica e androgênica. A Maca mostrou-se eficaz na redução de sintomas psicológicos, incluindo ansiedade e depressão, juntamente com a disfunção sexual associada à menopausa (GONZALES et al.,2014, DORDING et al. 2015, SIDDIQUI; BAIG; AHMAD, 2017).

Embora, existem poucos relatos de contraindicações, e de ser plantas autorizadas pela ANSIVA, não se pode concluir que ela é totalmente segura, 
demandando que sejam utilizadas de maneira racional, com orientação de um profissional capacitado. A Resolução nº 477/2008 do Conselho Federal de Farmácia, atribui ao profissional Farmacêutico, além de outras ações a promoção do uso racional de plantas medicinais, por meio de divulgação e comunicação aos pacientes, colaborando para que as pessoas façam o uso racional de plantas medicinais. A promoção do uso racional de ervas medicinais realizadas pelo Farmacêutico é uma prática importante, visto que utiliza-las de forma irracional acarreta danos à saúde dos indivíduos, pode propiciar um maior tempo de tratamento e cura, aumentar os custos, além de provocar interações medicamentosas negativas (RIBEIRO et al.,2013).

Nos estudos foi mostrado que a assistência farmacêutica consiste em uma atividade necessária se tratando dos serviços de atenção à saúde, já que traz como premissa a promoção do uso racional de medicamentos, agregando práticas que envolvem a escolha da terapia mais adequada, que garanta a menor ocorrência de efeitos adversos, avaliação das contraindicações existentes, garantia de que o paciente faça a adesão da terapia medicamentosa, o que garantirá, sobretudo, a segurança na utilização de plantas medicinais(FERNANDES; CEMBRANELLI,2015).

Desta maneira, Bastiani e colaboradores (2016), indicam a necessidade de que ao prestar a assistência farmacêutica, o profissional farmacêutico investigue se o paciente faz uso de algum outro medicamento, evitando que ocorram as interações medicamentosas negativas, que podem colaborar para a ocorrência da redução do efeito ou a potencialização do efeito fazendo com que a terapia medicamentosa não atinja o objetivo desejado.Foi destacada a importância da identificação de grupos de risco, é importante também verificar a prescrição feita pelo médico, com vistas a identificar a ocorrência de prescrição indevida e utilização inadequada. É atribuição do farmacêutico ainda, a orientação do paciente sobre o uso apropriado de plantas medicinais, os possíveis eventos adversos, sobretudo, quando o uso é realizado concomitantemente com outros fármacos (BATTISTI et al.,2013).

A Resolução no 546/20Ir disserta que o farmacêutico pode indicar plantas medicinais quando o paciente solicita, a indicação deverá ser feita de maneira clara, tendo registro em documento e uma via ser entregue ao paciente e a outra via deverá ficar no poder do farmacêutico. Com a Resolução citada, o farmacêutico tem na sua 
indicação pautada na prevenção de problemas ligados ao uso de medicamentos, a sensibilização do paciente em aderir à terapia medicamentosa, monitoramento e avaliação da resposta da terapia proposta, bem como a aproximação do farmacêutico na comunidade.

\section{CONSIDERAÇÕES FINAIS}

Com base nos estudos discutidos é possível concluir que a Tribulus terrestris consiste em uma planta com grandes potencialidades para o manejo da disfunção sexual e outros sintomas relacionados à menopausa, sendo assim, pode ser um tratamento alternativa ou complementar. Nos estudos foi mostrado que a Lepidium meyenii tem sido utilizada para aumentar a vitalidade, no estresse, na promoção da libido, no aumento da fertilidade e da performance sexual em homens e mulheres. Em homens a Maca aumenta o desejo sexual, a produção de espermatozóides e melhora a disfunção erétil de intensidade leve. Em mulheres reduz os sintomas de desconforto da menopausa, assim como melhora da disfunção sexual relacionada ao climatério.

\section{REFERÊNCIAS}

BARRETO, Ana Paula Pitiá et al. O impacto da disfunção sexual na qualidade de vida feminina: um estudo observacional. Revista Pesquisa em Fisioterapia, v. 8, n. 4, p. 5 II- $^{-}$ $517,2018$.

BATTISTI, Caroline et al. Plantas medicinais utilizadas no município de Palmeira das Missões, RS, Brasil. Revista Brasileira de Biociências, v. II, n. 3, 2013.

CHEN, Longfei; LI, Jieying; FAN, Liuping. The nutritional composition of maca in hypocotyls (Lepidium meyenii Walp.) cultivated in different regions of China. Journal of Food Quality, v. 2017, 2017.

DORDING, Christina M. et al. A double-blind placebo-controlled trial of maca root as treatment for antidepressant-induced sexual dysfunction in women. EvidenceBased Complementary and Alternative Medicine, v. 2015, 2015.

FERNANDES, Barbara Ferreira et al. Estudo etnofarmacológico das plantas medicinais com presença de saponinas e sua importância medicinal. Revista da Saúde da AJES, v. 5, n. 9, 2019. 
FERNANDES, Wendel Simões; CEMBRANELLI, Julio César. Automedicação e o uso irracional de medicamentos: o papel do profissional farmacêutico no combate a essas práticas. Revista Univap, v. 21, n. 37, p. 5-12, 2015.

GONZALES, Gustavo F et al. Maca (Lepidium meyenii Walp), una revisión sobre sus propiedades biológicas. Rev. perú. med. exp. salud publica, Lima , v. 3I, n. I, p. IOO-IIO, enero 2014 .

GUAZZELLI, Renata Mello et al. Estudo dos efeitos do Tribulus terrestris e da tibolona em mulheres com disfunção do desejo sexual após a menopausa/Study of the effects of Tribulus terrestris and tibolone in postmenopausal women with sexual desire dysfunction. Arquivos Médicos dos Hospitais e da Faculdade de Ciências Médicas da Santa Casa de São Paulo, p. 20-26, 2014.

QUEIROZ, Maria Amélia Crisóstomo et al. Representações sociais da sexualidade entre idosos. Revista brasileira de enfermagem, v. 68, p. 662-667, 2015.

RIBEIRO, Dayane Affonso. Estudo exploratório sobre a formação do profissional farmacêutico na área de plantas medicinais e fitoterápicos em universidades públicas e privadas do Estado do Rio de Janeiro. 2013. 39 f. Trabalho de Conclusão de Curso (Especialização) - Instituto de Tecnologia em Fármacos/Farmanguinhos, Fundação Oswaldo Cruz, Rio de Janeiro, 2013.

RIBEIRO, Jéssica Nunes et al. Disfunção Sexual Feminina. Revista Brasileira de Sexualidade Humana, v. 27, n. 2, 2016.

SANTOS, Mariana Alves et al. Sexualidade e aids na terceira idade: abordagem na consulta médica. Revista de Atenção à Saúde (ISSN 2359-4330), v. I5, n. 51, p. I8-22, 2017.

SIDDIQUI, Faheem Ahmed; BAIG, Iftikhar; AHMAD, Mansoor. Phytochemical and biological assessments on Lipidium meyenii (maca) and Epimidium sagittatum (horny goat weed). Pak. J. Pharm. Sci, v. 30, n. I, p. 029-036, 2017.

SILVA, Diego Augusto Lopes da. Efeitos do fitoterápico Tribulus terrestris sobre a próstata do gerbilo da Mongólia (Meriones unguiculatus): Effects of the herbal remedy Tribulus terrestris on the prostate of the Mongolian gerbil (Meriones unguiculatus). 2017. I recurso online (I64 p.). Tese (doutorado) - Universidade 
Estadual de Campinas, Instituto de Biologia, Campinas, SP.

SOUSA, Amanda Carvalho de; LIMA, Mizael Araujo. Tribulus terrestris Linn como tratamento da sintomatologia da menopausa: uma revisão sistemática. Revista Fitos, Rio de Janeiro, v. 13, n. 2, p. 195-203, 2019.

SOUZA, Karla Zanolla Dias. Avaliação dos efeitos do Tribulus terrestris em mulheres na pós-menopausa com distúrbio do desejo sexual hipoativo. 2015. I recurso online(5I p.). Dissertação(Mestrado)- Universidade Federal de Minas Gerais, Curso de Pós- Graduação em Ginecologia e Obstetrícia da Faculdade de Medicina, Minas Gerais .

WANG, Sunan; ZHU, Fan. Chemical composition and health effects of maca (Lepidium meyenii). Food chemistry, v. 288, p. 422-443, 2019.

ZENI, Ana Lúcia Bertarello et al. Utilização de plantas medicinais como remédio caseiro na Atenção Primária em Blumenau, Santa Catarina, Brasil. Ciência \& Saúde Coletiva, v. 22, p. 2703-2712, 2017. 